\title{
Retraction Note to: Development Strategy for Demand of ICT in Medium-Sized Enterprises of PRC
}

\author{
Yanhui Chen \\ School of Engineering, Linyi University, Linyi 276005, Shandong, P.R. China \\ cyh88790@126.com
}

M. Zhu (Ed.): ICCIC 2011, Part VI, CCIS 236, pp. 89-93, 2011.

(C) Springer-Verlag Berlin Heidelberg 2011

DOI 10.1007/978-3-642-24097-3_84

The paper starting on page 89 of this volume is an almost exact duplicate of the paper starting on page 84 . Both papers have been retracted. 